\title{
Removable of galvanic distortion on 3-D MT inversion.
}

\author{
Masanori Tani ${ }^{1}$, Hitoshi Mikada ${ }^{1}$, Tada-nori Goto ${ }^{1}$, Junichi Takekawa ${ }^{1}$ and \\ Weerachai Siripunvaraporn ${ }^{2}$ \\ 1 Kyoto University, \\ 2 Mahidol University
}

\begin{abstract}
Recent years, three-dimensional magnetotelluric (MT) inversion is widely used in geophysical structural surveys. In this paper, we show how to galvanic distortion would influence to 3-D MT inversion results, and would like to propose an improvement of model covariance matrix which could deal the galvanic distortion. For accommodating the galvanic distortion effects, we first simulate a set of synthetic MT response for some models and then add the distortion effects that are a function of observation locations. We applied WSINV3DMT as an inversion method to these synthetic MT responses. As a result, we confirmed distinct differences between 3-D MT inversion results depending on the degree of galvanic distortion. When we applied WSINV3DMT with the modified model covariance matrix, we obtained better inversion result. Based on these numerical experiments, we conclude that the galvanic distortion should be considered and be dealt with in the inversion.
\end{abstract}

\section{INTRODUCTION}

Recent years, 3-D MT inversions are widely used for geophysical investigations (e.g.Uchida and Sasaki, 2006).3-D MT inversion can get more realistic result than 2-D inversion, especially to the 3-D nature of geological structures, such as geothermal area or volcanic area (e.g.Siripunvaraporn, et al 2005b). Compered with 2-D MT inversions, 3-D MT inversions can recover more realistic and clear conductivity structure (Siripunvaraporn\& Egbert, 2000). Although the 3-D MT inversions have been applied to observed data recently, many refinements have not been considered. An effect of galvanic distortion on a 3-D MT inversion is the one of major problems. This research reveals the effects of galvanic distortion on 3-D MT inversion result and show an improved method of a 3-D MT inversion. The galvanic distortion is caused by local resistivity anomalies near the surface and makes artificial images in the inverted result model. Originally these effects of galvanic distortion should be reproduced as local anomaly at the surface layer model. But, because of the smoothness constraint, the galvanic distortion on MT response is reproduced as gradual resistivity change at deeper area especially for a resource exploration whose target depth are several kilometers. At those shallow depths, the effect of the galvanic distortion on inverted model would be strong as in the 2-D inversion, enough to get false anomaly in inverted model. For those reasons, the galvanic distortion has to be removed. In order to improve 3-D MT inversion results, we refine the smoothness function to be able to produce local anomaly on the surface layer of an inverted model. In this research, we modified a model covariance matrix in 3-D MT inversion algorithm, WSINV3DMT (Siripunvaraporn, et al., 2005a).

\section{METHOD}

\section{(1) The effect of galvanic distortion on} an inversion result model

We applied WSINV3DMT to several synthetic datasets to evaluate how local anomalies on the surface affects the result of 3-D MT inversion. The synthetic resistivity models used in this experiments are shown in Figure 1 and Figure2. The thickness of surface blocks is $10 \mathrm{~m}$. We estimated synthetic MT response functions from these two models for 7 piriods; $0.01 \mathrm{~s}, 0.05 \mathrm{~s}, 0.1 \mathrm{~s}, 0.5 \mathrm{~s}, 1 \mathrm{~s}, 5 \mathrm{~s}, 10 \mathrm{~s}$. Ant we applied WSINV3DMT to those synthetic datasets.
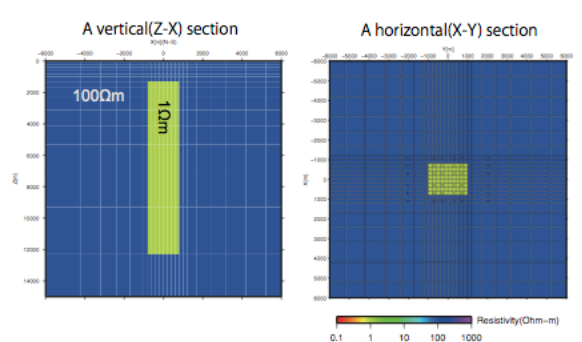

Figure 1. Underground resistivity structure for Models A and $\mathrm{B}$. Left figure is vertical sectioni at the center of the model. Right figure is horizontal slice which depth is $1300 \mathrm{~m}$ to $11500 \mathrm{~m}$ 

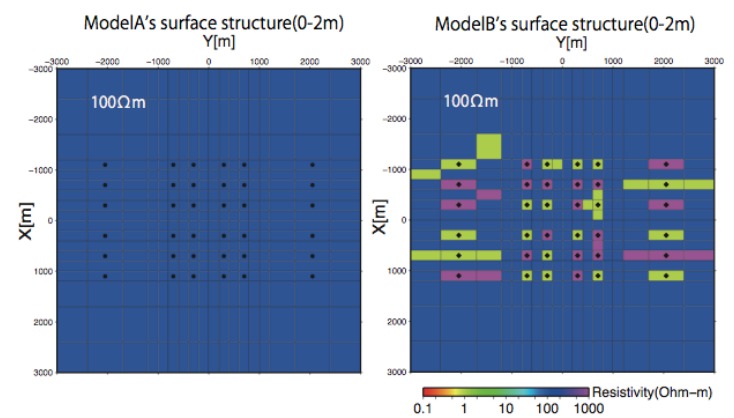

Figure 2. The surface resistivity structure for Models A and $\mathrm{B}$, black dots represent observation points.

\section{(2) Removable of the effect of galvanic distortion} on an inversion result model

We applied a modified model covariance matrix to MT response functions calculated from model B. In the modified method, we assume that effects of galvanic distortion can be expressed at the top thin layer. In WSINV3DMT which is based on Occam's inversion (Constable, Parker, \& Constable, 1987), the minimization function is described as follows:

$$
\begin{aligned}
U(\mathbf{m}, \lambda)= & \left(\mathbf{m}-\mathbf{m}_{0}\right)^{T} \mathbf{C}_{\mathbf{m}}^{-1}\left(\mathbf{m}-\mathbf{m}_{0}\right) \\
& +\lambda^{-1}(\mathbf{d}-\mathbf{F}[\mathbf{m}])^{T} \mathbf{C}_{\mathbf{d}}^{-1}(\mathbf{d}-\mathbf{F}[\mathbf{m}])
\end{aligned}
$$

where $\mathbf{m}$ is the resistivity model parameter, $\mathbf{m}_{\mathbf{0}}$ is the prior model parameter, $\mathbf{C m}$ is the model covariance matrix which defines the model norm, $d$ is the observed data, $\mathbf{F}[\mathbf{m}]$ is the model response, $\mathrm{Cd}$ is the data covariance matrix, and $\lambda^{-1}$ is a Lagrange multiplier. The model covariance matrix characterizes the smoothness of resistive variation relative to the base model. In order to express effects of galvanic distortion at surface layer in the inversion procedure models, we changed the model covariance matrix. In the new covariance matrix, the weighting of the smoothness between the first and second layers becomes smaller $(20 \%)$ than original one. Fig. 3 is a simple image of the modified model covariance.

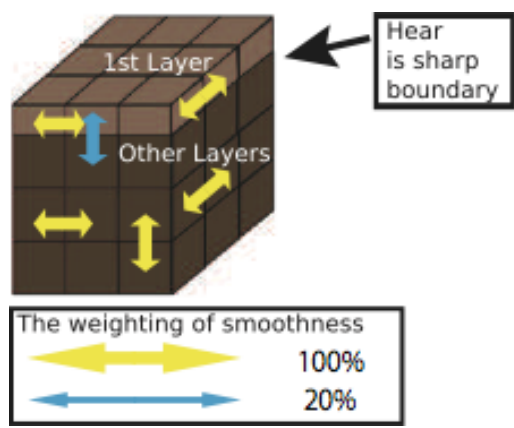

Figure 3. Explanation of modified weight for model covariance.

We apply WSINV3DMT with the modified model covariance matrix to another synthetic MT response.
In this examination, the synthetic model which is used to estimate synthetic observed MT response, is Model A (Figure2). First, we calculate MT response from the model we calculate synthetic distorted MT response $\left(\mathbf{Z}^{\text {obs }}\right)$, as follows. Finally we apply $3-D$ MT inversions(WSINV3DMT with original and modified covariance matrices). With the distortion matrix $\mathbf{C}$, we calculate $\mathbf{Z}^{\text {obs }}=\mathbf{C Z}$. This MT response $\mathbf{Z}^{\text {obs }}$ are synthetically distorted MT response. In this study, we determine $\mathbf{C}$ components range by results of forward calculation as shown Fig.4. We determine and add the various $\mathbf{C}$ to each observation points randomly. a)

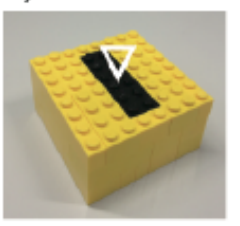

b)

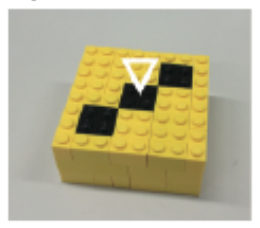

a)

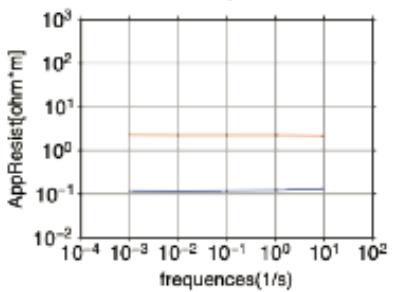

PHASE

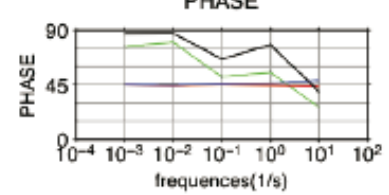

b)


c)
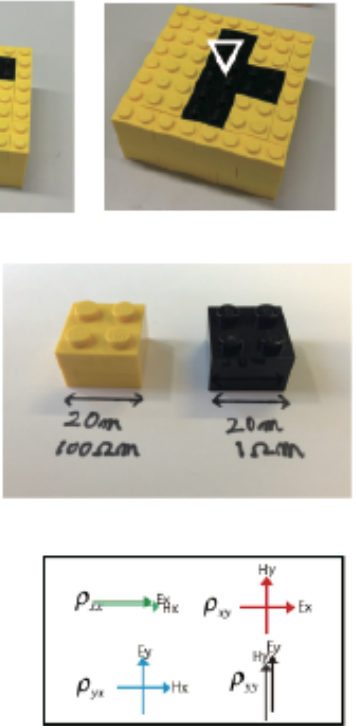

c)

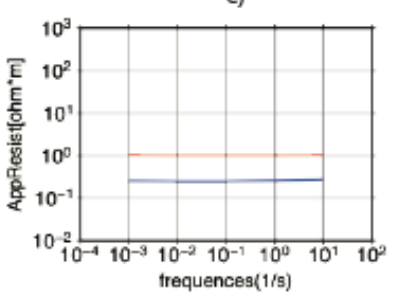

PHASE

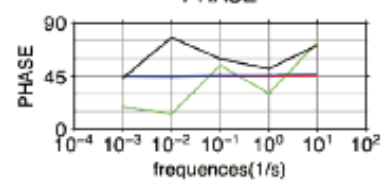

Figure 4. Examples of MT response calculated from homogeneous model with local anomaly on the surface. Block models represent near surface models from which distorted MT responses are calculated. From these MT responses we estimate conceivable ranges of distortion matrix $\mathbf{C}$.

\section{RESULTS AND DISCUSSION}

(1) The effect of galvanic distortion on an inversion result model.

Fig.5 and 6 show the inversion results(by WSINV3DMT with the conventional model 
covariance matrix) calculated from MT response with and without galvanic distortion, respectively (Models A and B). In Fig. 6, we can observe the artificial images due to the surface anomalies at various depth whereas the result in Figure 5 includes little artifacts. Fig. 7 is the inversion result from MT response with synthetic distortion matrix. This result also shows the effect of galvanic distortion clearly. Inversion algorithm fails to express local anomalies, and make false anomaly at deeper area.



Figure 5. Inversion result from MT response function on Model A by using the conventional model covariance matrix.

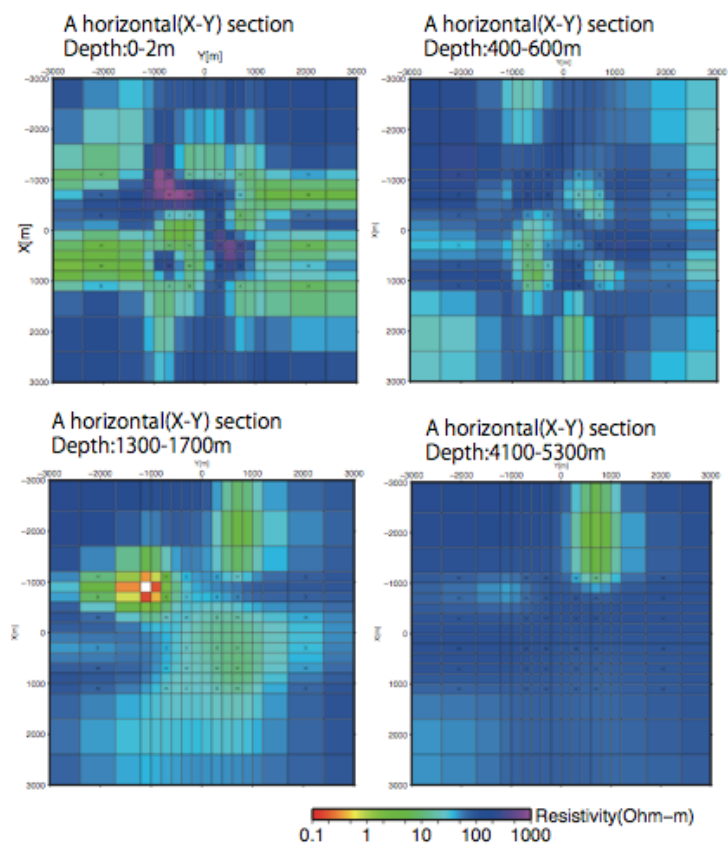

Figure 6. Inversion result from MT response function on Model B by using the conventional model covariance matrix.

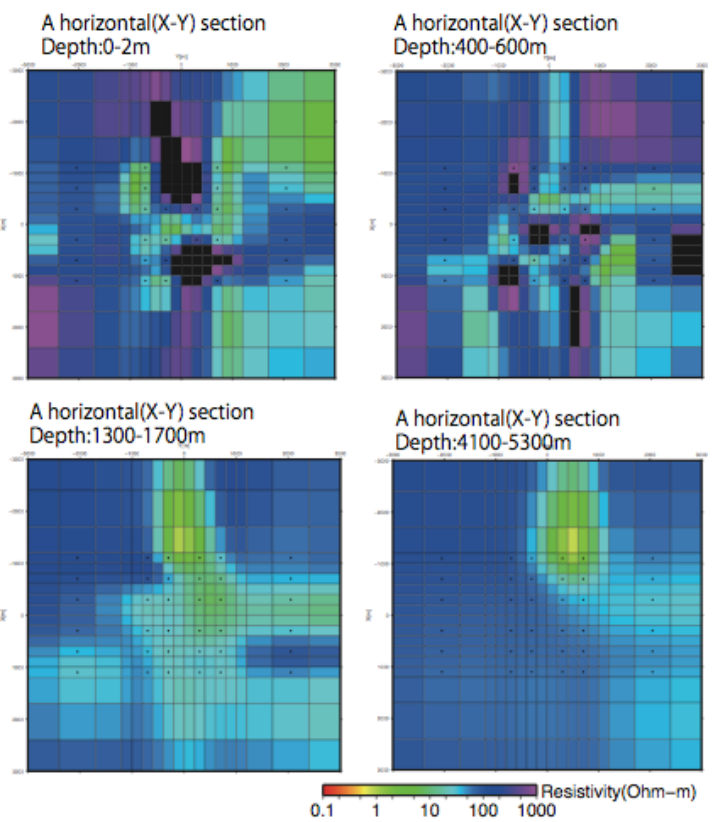

Figure 7. Inversion result, with distorted MT response function on Model A by using the conventional model covariance matrix.

(2) Removable of the effect of galvanic distortion on an inversion result model

Fig. 8 shows the inverted model of MT response function calculated from model $B$ with the new model covariance. In this result, the inverted resistivity structure model becomes more realistic compared with the model shown in Fig. 6 which is the inverted model from same datasets and with the ordinal model covariance. The galvanic distortion caused by local surface anomalies is recovered at the top layer and false anomalies at deeper area are removed. Fig. 9 is the new inversion result from MT response with synthetic distortion matrix. This result also shows better resistivity result than inversion result with original the model covariance matrix(Fig.7), especially in the deeper area. Although the modified model covariance indicates the effectiveness to the galvanic distortion, further discussion should be required. For example, we simply reduce the weight of the top layer in the smoothness calculation with $20 \%$ of original one. More suitable weighting should be discussed for avoiding too complex resistivity structure near the surface. 




Figure 8. Inversion result from MT response functioni on Model B with the modified model covariance matrix.



Figure 9. Inversion result(with modified model covariance matrix) from MT response function on Model A, distorted by synthetic distortion matrix.

\section{CONCLUSION}

In this paper, we first confirm the effects of galvanic distortion would generate false resistivity anomalies in the inverted model in particular in deeper part because of smoothness constraint. This problem should not be ignored to know realistic resistivity structure of the subsurface. The result of modified model covariance matrix is more reliable than that from the original model covariance matrix.
Since the thin surface layer could be analogues of the galvanic distortion, we could deal with the galvanic distortions by a thin layer at each of the observation locations.

\section{REFERENCES}

Constable, S., Parker, R., \& Constable, C. (1987). Occam's inversion: A practical algorithm for generating smooth models from electromagnetic sounding data. Geophysics, 52, 289-300.

Siripunvaraporn,W., \& Egbert, G. (2000, January). An efficient data-subspace inversion method for 2-D magnetotelluric data. Geophysics, 65 (3), 791-803.

Siripunvaraporn,W., Egbert, G., \& Uyeshima, M. (2005). Interpretation of two-dimensional magnetotelluric profile data with three-dimensional inversion: synthetic examples. Geophysical Journal International , 160 (3), 804-814.

Uchida, T., \& Sasaki, Y. (2006). Stable 3D inversion of MTdata and its application to geothermal exploration. Exploration GeoPhysics , 37 , 223-230. 